\title{
Discovery of Nodal Attributes through a Rank-Based Model of Network Structure
}

\author{
Adam Douglas Henry and Paweł Prałat
}

Abstract. The structure of many real-world networks coevolves with the attributes of individual network nodes. Thus, in empirical settings, it is often necessary to observe link structures as well as nodal attributes; however, it is sometimes the case that link structures are readily observed, whereas nodal attributes are difficult to measure. This paper investigates whether it is possible to assume a model of how networks coevolve with nodal attributes, and then apply this model to infer unobserved nodal attributes based on a known network structure. We find that it is possible to do so in the context of a previously studied "rank" model of network structure, where nodal attributes are represented by externally determined ranks. In particular, we show that node ranks may be reliably estimated by examining node degree in conjunction with the average degree of first- and higher-order neighbors.

\section{Introduction}

Research on complex networks has allowed us to better describe and model the interconnections among agents embedded within social, natural, and physical systems [Jackson 08, Newman 03, Wasserman and Faust 94]. Despite advances in

(C) Taylor \& Francis Group, LLC

ISSN: 1542-795I print 
the rigorous modeling of self-organizing networks (see, for example, [Bonato 08, Chung and $\mathrm{Lu} \mathrm{04]),} \mathrm{we} \mathrm{are} \mathrm{still} \mathrm{in} \mathrm{need} \mathrm{of} \mathrm{better} \mathrm{models} \mathrm{of} \mathrm{how} \mathrm{nodal} \mathrm{attributes}$ both influence and coevolve with network structure. Such models are crucial, given the well-known role of nodal attributes in driving link structure. In the web graph, for instance, it has been posited that new linkages are formed with probability proportional to node degree [Barabási and Albert 99]. However, it is also likely that link formation is determined by node-level variables. For example, through homophily processes, linkages may form with higher probability if two webpages have very similar lexical content (one possible application of a "spatial" preferential attachment model; see [Aiello 09, Janssen et al. 10]). Models that explicitly account for the interplay between link structures and nodal attributes are therefore relevant to research on the web graph, but also apply more broadly to the study of complex social networks [Lazer 01].

In this paper, we investigate how models of complex networks may be used to reliably estimate nodal attributes based on observations of network structure alone. Given the importance of nodal attributes in driving the dynamics of many real-world systems, it is important to understand how we may estimate such variables when empirical observations are difficult or impossible. For example, in some social networks (such as online social networks), it may be quite easy to observe interactions, but the actual prestige, beliefs, or emotions of individuals within the network (which may partly determine or be determined by structure) are not easily measured. This research illustrates how one may create a mathematical model of the coevolution of network structure and nodal attributes and then "reverse" this model to develop reliable estimates of individual nodal attributes based on knowledge of the link structure only.

We illustrate this approach using an existing model of network selforganization proposed in [Janssen and Prałat 09], which in turn is a generalization of the model proposed in [Euczak and Prałat 06]. This model fits well into the larger enterprise of modeling the coevolution of networks and nodal attributes, illustrated, for example, in [Henry et al. 11, Janssen and Prałat 09, Janssen and Prałat 10, Łuczak and Prałat 06, Prałat 08, Prałat 11, Prałat and Wormald 09]. In the model used here, linkages are chosen based in part on nodes' externally determined rank relative to other nodes. Use of this ranking scheme allows us to model attributes that change stochastically as nodes enter and exit the system over time. In doing so, we build on prior work on ranking models [Fortunato et al. 06, Janssen and Prałat 09, Luczak and Prałat 06] and suggest some new directions for future research and applications of these models.

Our paper builds on earlier research presented at the 2010 Workshop on Algorithms and Models for the Web Graph (WAW) conference [Henry and Prałat 11]. As with the earlier paper, our central purpose is to illustrate the feasibility of 
reversing mathematical models of network self-organization to estimate nodal attributes. We investigate this core question in the context of ranking models by showing how one can reliably infer the externally determined rank of nodes based on observations of node degree and the average degree of first-order neighbors, the average degree of second-order neighbors, and so on. While we originally illustrated the possibility of inferring ranks based on observed degree through computational simulation only, this paper demonstrates how one may rigorously infer node attributes based on structural properties alone. We turn first to a discussion of the ranking model. We then consider the problem of inferring ranks based on observations of node degree and suggest some areas for future research.

\section{Model}

In this section, we formally define a ranking model that reflects the attributes of nodes within a hypothetical system. This model, called the protean graph, was introduced in [Euczak and Prałat 06] and then generalized to other ranking schemes in [Janssen and Prałat 09]. This model not only specifies the process by which attributes are assigned to individual nodes, but also specifies the way in which these attributes shift over time as nodes enter or exit the system. The model focuses on systems in which the total number of nodes is large but fixed (for example, if at each time step, a node is removed uniformly at random and immediately replaced by a new one). This type of behavior is most consistent for well-established systems. Such stochastic systems are also usually more challenging to model than, say, systems that are "young" or "middle-aged" and hence growing over time, with nodes being added to the system at a faster rate than they are removed.

The only modification in the model we study in this paper from the model studied in [Janssen and Prałat 09] is that an initial rank is biased toward average values. However, the proofs in [Janssen and Prałat 09] may be easily adjusted to the new ranking function used here. We omit proofs in this paper, stating the results only. The second minor difference is that in [Janssen and Prałat 09], the model was defined as a graph process, whereas in this paper, the model is split into two parts. In the first part, we focus on the behavior of the rank function only, and in the second part, we introduce a graph process overlaid on the rank function. Separating these two pieces of the model aids tractability and illustrates the independence of the ranking and graph evolution processes.

All results presented here are asymptotic (that is, with $n$ tending to infinity). We say that an event holds asymptotically almost surely if it holds with probability tending to 1 as $n \rightarrow \infty$. We will sometimes use the stronger notion of 
"with extreme probability" in favor of the more commonly used "asymptotically almost surely," since it simplifies some of our proofs. We say that an event holds with extreme probability if it holds with probability at least $1-\exp \left(-\Theta\left(\log ^{2} n\right)\right)$ as $n \rightarrow \infty$. Thus, if we consider a polynomial number of events each of which holds with extreme probability, then all events hold with extreme probability. To combine this notion with asymptotic notation such as $O()$ and $o($ ), we follow the conventions in [Wormald 04].

\section{I. A Rank Model of Content}

At each time $t$, we have exactly $n$ objects (such as nodes, which may represent agents in a social system) in a set $V_{t}$. Moreover, at each time $t$, each object $v \in V_{t}$ has rank $r_{t}(v) \in[n]$ (we use $[n]$ to denote the set $\{1,2, \ldots, n\}$ ). In order to obtain a proper ranking, the rank function $r_{t}: V_{t} \rightarrow[n]$ is a bijection for all $t$, so every object has a unique rank. In agreement with the common use of the word "rank," high rank refers to an object $v$ for which $r_{t}(v)$ is small: the highest-ranked object is ranked number one, so has rank equal to 1; the lowest-ranked object has rank $n$. The initialization and update of the ranking is done according to a so-called ranking scheme. Various ranking schemes may be considered, and each might lead to different behaviors. We first define the general model, and then list a few natural ranking schemes.

The model produces a sequence $\left\{\left(V_{t}, r_{t}\right)\right\}_{t=0}^{\infty}$ of sets $V_{t}$ of $n$ objects and ranking functions $r_{t}$, where $t$ denotes time. To initialize the model, let $V_{0}$ be any set of $n$ objects and let $r_{0}$ be any initial rank function $r_{0}: V_{0} \rightarrow[n]$ that is consistent with the ranking scheme. For $t \geq 1$, we form $\left(V_{t}, r_{t}\right)$ from $\left(V_{t-1}, r_{t-1}\right)$ according to the following rules:

(i) Choose uniformly at random an object $u_{t} \in V_{t-1}$ and delete it.

(ii) Add a new object $v$. (We refer to the time step $t$ in which object $v$ was added as the time at which $v$ was born.)

(iii) Assign an initial rank to $v$ and update $V_{t}$ and the ranking function $r_{t}: V_{t} \rightarrow[n]$ according to the ranking scheme.

One can define a number of different ranking schemes. In this paper, we focus on the random initial rank scheme, but the concept of ranking by age will also be important. Therefore, let us define the following two schemes. In order to distinguish them, we will use $a_{t}$ for the ranking by age and $r_{t}$ for the random initial rank. 
Ranking by age. The newly added object $v$ obtains an initial rank $n$; its rank decreases by 1 each time an object with smaller rank is removed. Formally, for each $v \in V_{t-1} \backslash\left\{u_{t}\right\}$, we have $a_{t}(v)=a_{t-1}(v)-\gamma$, where $\gamma=1$ if the rank of the object deleted in step $t$ is smaller than $a_{t-1}(v)$, and $a_{t}(v)=0$ otherwise.

Random initial rank. The object added at time $t$ obtains an initial rank $R_{t}$ that is randomly chosen from $[n]$ according to a prescribed distribution. Ranks of all objects are adjusted accordingly. Formally, for each $v \in V_{t-1} \backslash\left\{u_{t}\right\}$, we have $r_{t}(v)=r_{t-1}(v)+\delta-\gamma$, where $\delta=1$ if $r_{t-1}(v)>R_{t}$, while $\delta=0$ otherwise, and $\gamma=1$ if the rank of $u_{t}$, the object deleted in step $t$, is smaller than $r_{t-1}(v)$, and $\gamma=0$ otherwise.

The coupon collector problem can give us insight into when all objects from the initial set $V_{0}$ will be deleted. Namely, let $L=n(\log n+\omega(n))$, where $\omega(n)$ is any function tending to infinity with $n$. It is a well-known result that asymptotically almost surely, after $L$ steps, all original objects will have been deleted.

The behavior of the age-rank function $a_{t}(v)$ was studied in [Janssen and Prałat 09].

Theorem 2.I. [Janssen and Prałat 09] Let $a_{t}(v)$ be the age rank of object $v$ at time $t$. Then with extreme probability, for every $t$ in the range

$$
0 \leq t \leq t_{f}=\frac{1}{2} n \log n-2 n \log \log n
$$

we have

$$
a_{t}(v)=n \exp \left(-\frac{t}{n}\right)\left(1+O\left(\log ^{-1 / 2} n\right)\right),
$$

conditional on the object $v$ surviving until time $t_{f}$.

We consider the case in which the rank $R_{i}$ of the object $v$ added at time $i$ is chosen at random from $[n]$. The ranks of existing objects are adjusted accordingly. We make the assumption that all initial ranks are chosen according to the same distribution. In particular, we fix a continuous bijective function $F:[0,1] \rightarrow$ $[0,1]$, and for all integers $1 \leq k \leq n$, we let

$$
\mathbb{P}\left(R_{i} \leq k\right)=F\left(\frac{k}{n}\right) .
$$

Thus, $F$ represents the limit, as $n$ goes to infinity, of the cumulative distribution functions of the variables $R_{i}$. To simplify the calculations while exploring a 
wide array of possibilities for $F$, we assume $F$ to be of the form

$$
F(x)= \begin{cases}(2 x)^{s} / 2 & \text { if } 0 \leq x \leq 1 / 2 \\ 1-(2(1-x))^{s} / 2 & \text { if } 1 / 2<x \leq 1\end{cases}
$$

where $s \geq 1$. In [Janssen and Prałat 09], a similar function was analyzed, namely $F(x)=x^{s}$. We modify the function slightly to obtain the behavior we desire, namely, that average initial ranks occur more often than both extreme cases. As noted earlier, the proofs are easily adapted to a new function.

This distribution has the advantage of allowing us to generalize our results to a broad class of realistic initial distributions, ranging from situations in which initial ranks are distributed uniformly at random (when $s=1$ ) to situations in which agents enter the system with a mediocre rank with higher probability (when $s>1$; in this case, the highest probability rank is $n / 2$ ). For example, Figure 1 shows the differences in distributions across $s=1.0, s=1.2$, and $s=$ 1.5. This functional form is reasonable, because it reflects the notion that many types of attributes follow a normal distribution in social systems; it tends to be unlikely that new agents will be "born" into the system with a very low rank or a very high rank. If rank represents a type of dynamic fitness whereby agents compete for better ranks, entering agents are unlikely to have poor ranks, because then they may not be able to enter the system at all, and they are also unlikely to enter with very good ranks, which are obtained only through a

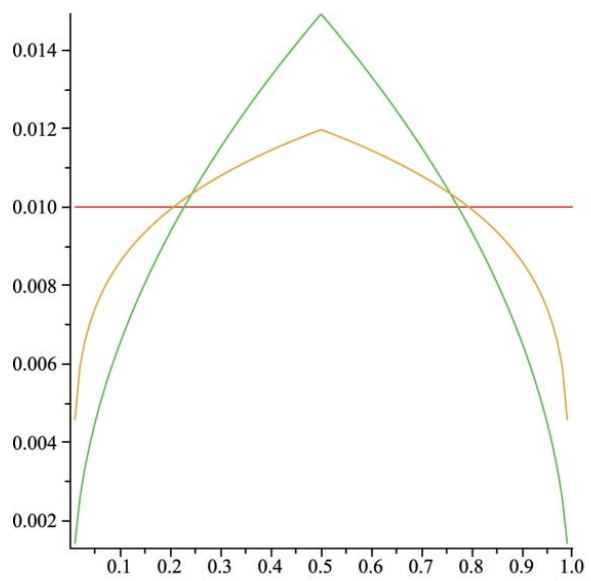

(a) $f(x)=\mathbb{P}\left(x \leq R / n \leq x+\frac{1}{100}\right)$

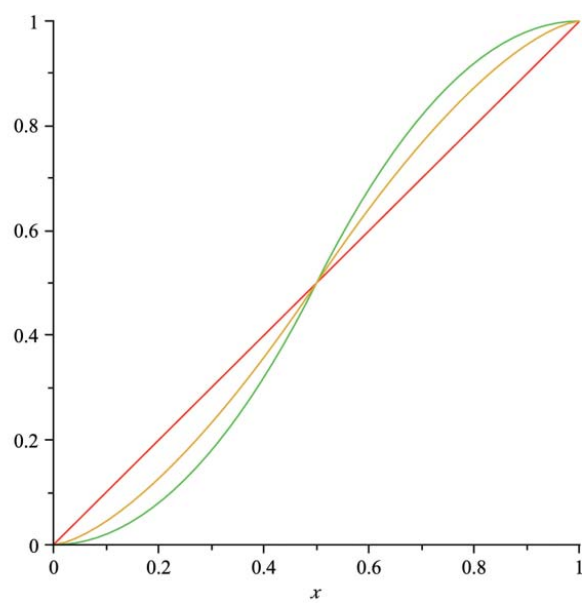

(b) $F(x)=\mathbb{P}(R / n \leq x)$

Figure I. Different distributions: $s=1.0,1.2,1.5$ (color figure available online). 
history of competition in the system. Our functional form for $F(x)$ reflects these possibilities.

The case $s=1$ represents the uniform distribution of the $R_{i}$. The random variable $r_{t}(v)$ is sharply concentrated around the initial rank $R_{i}$. The following result was obtained in [Janssen and Prałat 09].

Lemma 2.2. Suppose that object $v$ obtained an initial rank $R \geq \sqrt{n} \log ^{2} n$. Then with extreme probability,

$$
r_{t}(v)=R\left(1+O\left(\log ^{-1 / 2} n\right)\right)
$$

to the end of its life.

In the case $s>1$, the initial rank is biased toward the midrange ranks. The rank function exhibits more-complex behavior in this case. Due to the symmetry of the function $F(x)$, without loss of generality we can assume that an initial rank is at most $n / 2$. For ranks close to $n / 2$ we clearly cannot predict the behavior; the final rank can be bigger or smaller than the initial rank. However, if the initial rank is separated a bit from the middle rank, then we get a concentration.

Theorem 2.3. Suppose that an object $v$ obtained an initial rank

$$
r_{0}(v)=R<\frac{n}{2}-\sqrt{n} \log ^{2} n
$$

at time 0 . Then with extreme probability, for every $t$ in the range

$$
0 \leq t \leq t_{f}=\frac{1}{2} n \log n-2 n \log \log n,
$$

conditional upon the object $v$ surviving until time $t$,

$$
r_{t}(v)=\frac{n}{2}\left(\left(\left(\frac{2 R}{n}\right)^{1-s}-1\right) e^{(s-1) t / n}+1\right)^{1 /(1-s)}\left(1+O\left(\log ^{-1 / 2} n\right)\right)
$$

provided

$$
\frac{n}{2}\left(\left(\left(\frac{2 R}{n}\right)^{1-s}-1\right) e^{(s-1) t / n}+1\right)^{1 /(1-s)} \geq \sqrt{n} \log ^{2} n
$$

Figure 2 presents the behavior of different initial ranks for one specific value of $s=1.2$ as well as the behavior of one specific initial rank, $R=0.4 n$, for different values of $s$. Both rank and time are scaled by $n$. 


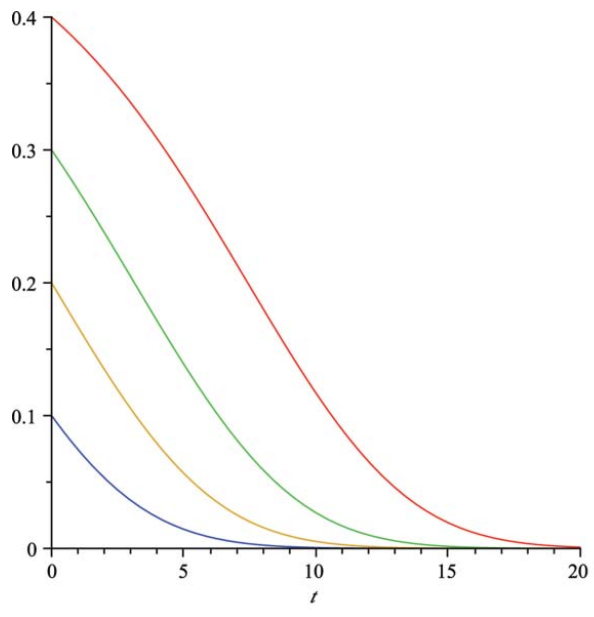

(a) $R=0.1,0.2,0.3,0.4$

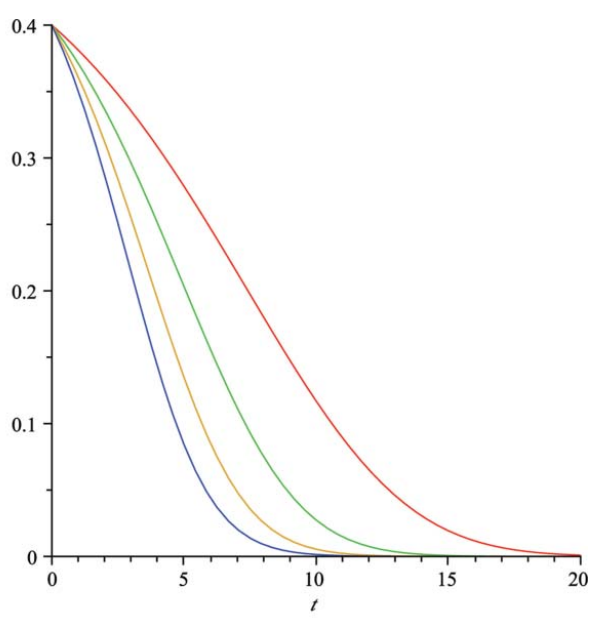

(b) $s=1.2,1.3,1.4,1.5$

Figure 2. (a) The behavior for different initial ranks $(s=1.2)$. (b) The behavior for different values of $s(R=0.4)$ (color figure available online).

\subsection{A Rank Model of Network Structure}

In this subsection, we introduce the network on top of the process discussed in previous sections. We need two more parameters: the attachment strength $\alpha \in(0,1)$ and initial degree $d \in \mathbb{N}$. This time, the model produces a sequence $\left\{\left(G_{t}, r_{t}\right)\right\}_{t=0}^{\infty}$ of graphs $G_{t}=\left(V_{t}, E_{t}\right)$ on $n$ vertices and ranking functions $r_{t}$ : $V_{t} \rightarrow[n]$. To initialize the model, let $G_{0}$ be any graph on $n$ vertices and let $r_{0}$ be any initial rank function $r_{0}: V_{0} \rightarrow[n]$ that is consistent with the ranking scheme. For $t \geq 1$, we form $G_{t}$ from $G_{t-1}$ according to the following rules:

(i) Choose uniformly at random a vertex $u_{t} \in V_{t-1}$ and delete it.

(ii) Add a new vertex $v_{t}$ together with $d$ edges from $v_{t}$ to existing vertices chosen randomly with weighted probabilities. The edges are added in $d$ substeps. In each substep, one edge is added, and the probability that $v_{i}$ is chosen as its endpoint (the link probability) is proportional to $r_{t-1}\left(v_{i}\right)^{-\alpha}$.

(iii) Assign an initial rank to $v_{t}$ and update $V_{t}$ and the ranking function $r_{t}: V_{t} \rightarrow[n]$ according to the ranking scheme.

At this point we must wait until time $L$, when all vertices from the initial graph $G_{0}$ are deleted. At this point, we reach the stationary distribution, and 
our task is to analyze the behavior of a random graph $G_{L}$. As noted previously, $L \leq n(\log n+\omega(n))$ asymptotically almost surely, where $\omega(n)$ is any function tending to infinity with $n$.

In [Janssen and Prałat 09], it was shown that the uniform distribution for the initial rank (that is, the specific case of $s=1$ in our model) generates a powerlaw degree distribution with exponent $1+1 / \alpha$. One can adapt the proofs from [Janssen and Prałat 09] and show that this is also the case for $s>1$.

\section{The Discovery of Nodal Attributes through Structure}

While the web graph is a useful platform for social network research, the notion that networks self-organize as a function of nodal attributes suggests the need to observe both the structure and attributes of the nodes. While structures may be observed directly, for example through hyperlink data, in many cases, attributes (ranks) of agents embedded in the network will be latent (unobserved) variables. However, given a realistic model of the process by which the network was generated, it is possible to infer likely attributes of nodes. Here we begin with a heuristic argument for how nodal attributes may be inferred from structure, followed by a more rigorous analysis of this problem. ${ }^{1}$

\section{I. A Heuristic Argument}

We focus on time $L$, when all vertices from the initial graph $G_{0}$ have been deleted and the stationary distribution of the Markov chain is reached. Consider the degree $\operatorname{deg}(v, L)$ of a given node. In this section, let us for simplicity focus on "in-degree" $\operatorname{deg}^{-}(v, L)$ of $v$, that is, the number of neighbors of $v$ that are older than this vertex.

Note that for a vertex of high degree, the number of younger neighbors is negligible compared to the total degree. Moreover, despite the fact that precise ages of vertices are difficult to estimate in practice, given any edge $u w$, it is known which vertex ( $u$ or $w)$ is older. Thus, it makes sense to focus on in-degrees here.

Given the model outlined here, this degree (or in-degree) is a function of two factors related to content: first, the length of time the node has been in the system, and second, the initial rank assigned to the node when it was born into the network. Agents with smaller initial ranks tend to have larger degrees, and older vertices also tend to have larger degrees. Despite this correlation, the

\footnotetext{
${ }^{1}$ The simulations presented in the paper were performed using a program written in $\mathrm{C} / \mathrm{C}++$, which may be found at http://www.math.ryerson.ca/ pralat/.
} 

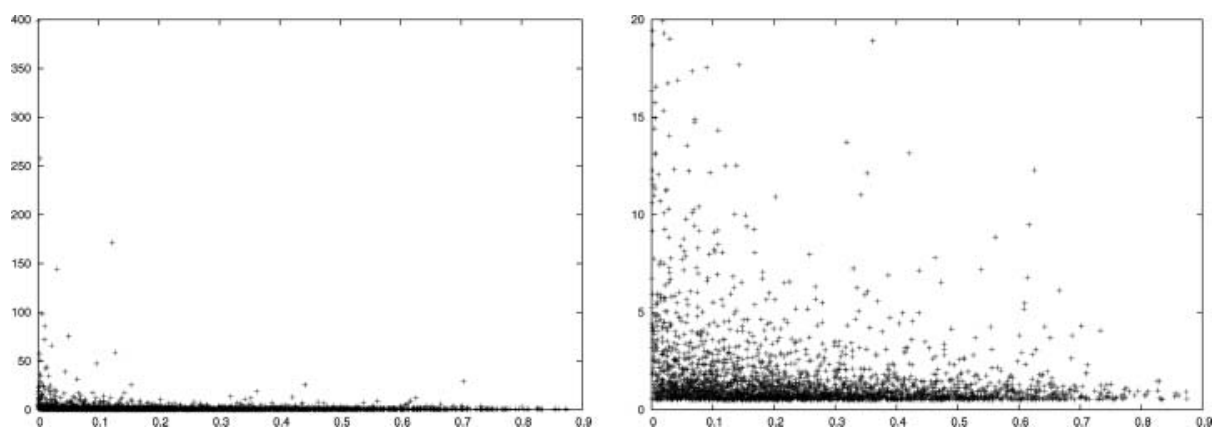

Figure 3. In-degree of $v$ vs. the age rank of $v$ (rescaled)

true relationship is quite complicated, and it would seem to be a lost cause to try to infer only one of these attributes (age or rank) based on degree only. Figure 3 presents the relationship between age and degree for vertices of degree at least $d / 2$ when networks are simulated according to the model described here $(n=20000, d=100, s=1.5$, and $\alpha=0.8)$. Young vertices have small degree (there is no time to accumulate neighbors, even if the initial rank is good), but old vertices can still have small degree (because they have an unattractive rank).

It turns out, however, that it becomes feasible to estimate these properties when we broaden our focus from the degree of a single agent to properties of their second neighborhood. Consider, for example, the following coefficient defined for a vertex $v$ of nonzero degree that is proportional to the average degree of neighbors of $v$ :

$$
b_{2}(v)=\frac{\sum_{u \sim v} \operatorname{deg}^{-}(u)}{\operatorname{deg}^{-}(v)} .
$$

We put $b_{2}(v)=0$ if $\operatorname{deg}^{-}(v)=0$. Clearly, old nodes have more old neighbors compared to younger nodes. In other words, there is a correlation between the age of $v$ and ages of its neighbors. On the other hand, ranks are generated independently, so a distribution of ranks of the neighbors of $v$ should be similar to the distribution we use in the model. The more neighbors $v$ has, the greater the correlation should be. Older vertices should have larger $b_{2}(v)$ coefficients. See Figure 4(a) for the relation for vertices of degree at least $d / 2$.

This process can be carried even further to develop more finely tuned estimates of nodes' unobserved attributes. We can take a look at third-, fourth-, and higherorder neighborhoods by defining, recursively, for $k \geq 3$,

$$
b_{k}(v)=\frac{\sum_{u \sim v} b_{k-1}(u)}{\operatorname{deg}^{-}(v)},
$$




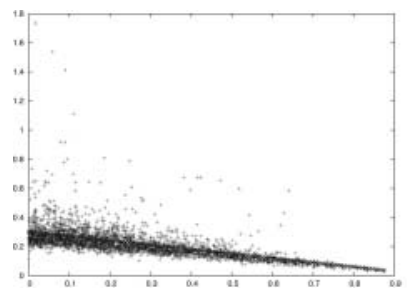

(a) $b_{2}(x)$

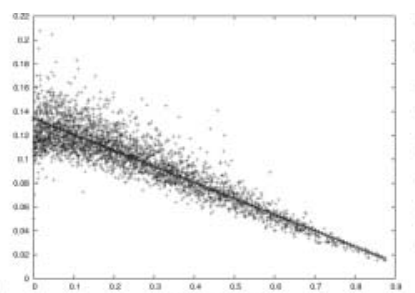

(b) $b_{3}(x)$

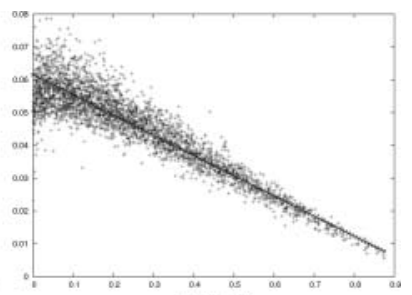

(c) $b_{4}(x)$

Figure 4. $b_{k}(x)$ vs. the age rank of $v$ (rescaled).

provided that $\operatorname{deg}^{-}(v)>0$; otherwise, $b_{k}(v)=0$. Again, in this case, older vertices should have larger coefficients, and the error should decrease for, say, $k=3$ and $k=4$. See Figure 4(b), (c) for the results for $b_{3}(x)$ and $b_{4}(x)$.

Even on casual examination, these scatterplots reveal a strong, nearly linear, relationship between the average degree of neighbors (or higher-order $b_{k}$ coefficients) and the age of a node. Inferring rank rather than age may be accomplished in a similar way.

One way of viewing the increasing predictive power of these structural characteristics is to perform a simple OLS linear regression with node age as the dependent variable (the unobserved variable to be inferred in real-world applications) and degree or various $b_{k}$ measures as possible independent variables. Using this approach, we find that predicting age as a linear function of average degree becomes more precise as we move to higher-order neighborhoods. For example, the $R^{2}$ statistic when age is predicted using degree is only 0.01 , meaning that node degree explains only $1 \%$ of the variance in actual node age. When the average degree of a neighbor $\left(b_{2}\right)$ is used as an independent variable, a linear model explains $35 \%$ of the variance on age $\left(R^{2}=0.35\right)$. For $b_{3}, R^{2}$ jumps to 0.77 , and to 0.83 for $b_{4}$.

These regression models provide at least heuristic evidence that one can achieve fairly accurate predictions of age when one examines the degree of neighbors, and neighbors of neighbors, and so forth. And while these linear models are suggestive of strong patterns, the scatterplots also make it clear that the accuracy with which we can predict age depends on the degree of the node. In particular, it seems that for low-degree nodes, age may be predicted with fair accuracy (in particular, because a node having just entered the system, the number of relationships is a more direct result of initial rank) while the relationship between $b_{k}$ and age for high-degree nodes is less precise.

It is also interesting to note that going from the second neighborhood to the third neighborhood provides a smaller marginal benefit in terms of predictive power, as measured by the $R^{2}$ values. While examination of the third 
neighborhood provides the strongest inferences regarding age, of course there will be an upper bound on the depth of neighborhoods that may be examined, plus there is likely to be an optimal neighborhood to examine in terms of maximizing the predictive power of this method. These issues, along with the strength of predictions that may be made for small- versus high-degree agents, are discussed presently.

\subsection{A Rigorous Approach to Inferring Nodal Attributes}

In order to obtain an estimate for $b_{2}(v)$ (or $b_{k}(v)$ in general), we need to calculate the expected (in- and total) degree of a vertex $v_{i}$ with age rank $a_{L}\left(v_{i}\right)=i=x n$. In the proof of Theorem 5.6 (see the appendix, Section 5), we provide a useful estimate for the case in which the expected value tends to infinity together with $n$. This was enough to prove that the degree distribution follows a power law, despite the fact that almost all vertices have a constant expected degree. When the expected degree does not grow with $n$, we cannot, of course, expect the degree to be well concentrated around this value. However, when a large number of vertices is considered, the average degree must be very close to what we expect (by the Chernoff bound). Hence, if the degree of a vertex $v$ (for which we aim to have a well-estimated parameter) is large enough, then the value of $b_{2}(v)$ is well concentrated (recall that $b_{2}(v)$ is, in fact, an average degree of a neighbor of $v$ ).

Suppose that the initial rank of $v_{i}$ is at most $n / 2-\sqrt{n} \log ^{2} n$. A more careful estimate than the one used in Theorem 5.6 will give us an asymptotic value of the expected degree of $v_{i}$ at time $L$. To get a better estimate for the number of initial neighbors of $v_{i}$ that are not deleted at time $L$, we note that $i-1$ vertices are older than $v_{i}$, and hence we expect that $v_{i}$ has

$$
\frac{i-1}{n-1} d=(1+o(1)) x d
$$

older neighbors. Therefore,

$\mathbb{E} \operatorname{deg}\left(v_{i}, L\right) \sim x d+d(1-\alpha) 2^{\alpha} \int_{x}^{1}\left(\left(\left(\frac{2 R}{n}\right)^{1-s}-1\right)\left(\frac{y}{x}\right)^{s-1}+1\right)^{-\alpha /(1-s)} d y$

where the notation $a \sim b$ stands for $a=(1+o(1)) b$. In the formula for $\operatorname{deg}^{-}\left(v_{i}, L\right)$, there is no $x d$ term. The rest remains exactly the same.

Suppose now that the initial rank of $v_{i}$ is at least $n / 2+\sqrt{n} \log ^{2} n$. Due to the symmetry of ranks, the rank of $v_{i}$ behaves exactly like $n-r_{t}\left(v_{i}^{*}\right)$, where $v_{i}^{*}$ is a vertex that obtained the initial rank of $n-R \leq n / 2-\sqrt{n} \log ^{2} n$. Using Theorem 
2.3 as before, we get that with extreme probability, $v_{i}$ had rank

$$
r_{t_{j}}\left(v_{i}\right) \sim n-\frac{n}{2}\left(\left(\left(\frac{2(n-R)}{n}\right)^{1-s}-1\right)\left(\frac{y}{x}\right)^{s-1}+1\right)^{1 /(1-s)},
$$

when $v_{j}(j=y n)$ was born, and so

$$
\begin{aligned}
& \mathbb{E} \operatorname{deg}\left(v_{i}, L\right) \sim x d \\
& +d(1-\alpha) \int_{x}^{1}\left(1-\frac{1}{2}\left(\left(\left(\frac{2(n-R)}{n}\right)^{1-s}-1\right)\left(\frac{y}{x}\right)^{s-1}+1\right)^{1 /(1-s)}\right)^{-\alpha} d y .
\end{aligned}
$$

(Again in the formula for $\operatorname{deg}^{-}\left(v_{i}, L\right)$, there is no $x d$ term; the rest remains exactly the same.) Since $\mathbb{P}(R \leq z n)=F(z)=(2 z)^{s} / 2$ for $z \leq 1 / 2$ and $F(z)=$ $1-F(1-z)$, we get immediately the following result. Let

$$
\begin{aligned}
g(x)= & d(1-\alpha) s 2^{\alpha+s-1} \int_{0}^{1 / 2} z^{s-1} \int_{x}^{1}\left(\left((2 z)^{1-s}-1\right)\left(\frac{y}{x}\right)^{s-1}+1\right)^{-\alpha /(1-s)} d y d z \\
+ & d(1-\alpha) s 2^{s-1} \\
& \times \int_{0}^{1 / 2} z^{s-1} \int_{x}^{1}\left(1-\frac{1}{2}\left(\left((2 z)^{1-s}-1\right)\left(\frac{y}{x}\right)^{s-1}+1\right)^{1 /(1-s)}\right)^{-\alpha} d y d z .
\end{aligned}
$$

Theorem 3.I. Let $0<\alpha<1, d \in \mathbb{N}, i=i(n) \in[n]$, and let $v_{i}$ be the vertex whose age rank at time $L$ equals $a_{L}\left(v_{i}\right)=i=x n$. Then the expected (in-)degree of $v_{i}$ is given by

$$
\mathbb{E} \operatorname{deg}\left(v_{i}, L\right) \sim x d+g(x), \quad \mathbb{E} \operatorname{deg}^{-}\left(v_{i}, L\right) \sim g(x)
$$

It appears that there are no closed formulas for $\mathbb{E} \operatorname{deg}\left(v_{i}, L\right)$ and $\mathbb{E} \operatorname{deg}^{-}\left(v_{i}, L\right)$, but these values may easily be estimated numerically. Figure $5(\mathrm{a})$, (b) presents the expected degree for the set of parameters used before (that is, $d=100$, $s=1.5$, and $\alpha=0.8) .^{2}$

Let us note that the behavior we experience here is similar to that for the protean graph [Euczak and Prałat 06], which may be viewed as the father of rank-based models. Old vertices have very large expected value, since they have nonnegligible probability of getting small initial rank (which implies a good rank during the whole process and thus large expected degree). Young vertices still

\footnotetext{
${ }^{2}$ The computations presented in this paper were performed using Maple. The worksheets may be found at http://www.math.ryerson.ca/ pralat/.
} 


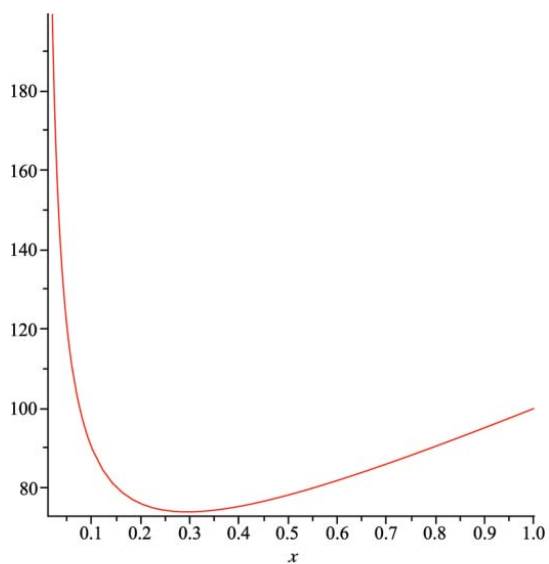

(a) $x d+g(x)\left(\mathbb{E} \operatorname{deg}\left(v_{i}, L\right)\right)$

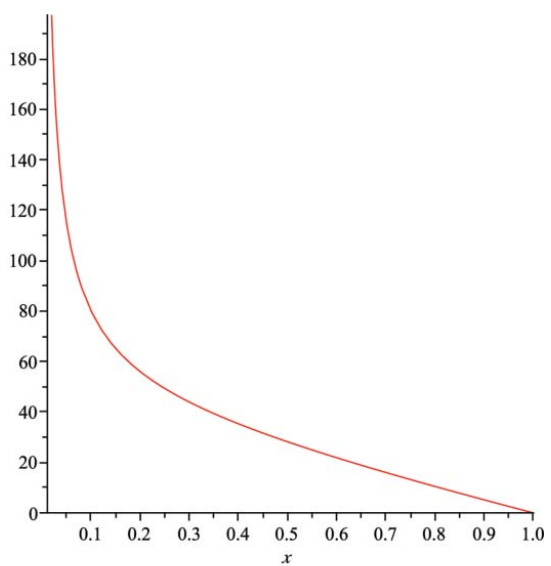

(b) $g(x)\left(\mathbb{E} \operatorname{deg}^{-}\left(v_{i}, L\right)\right)$

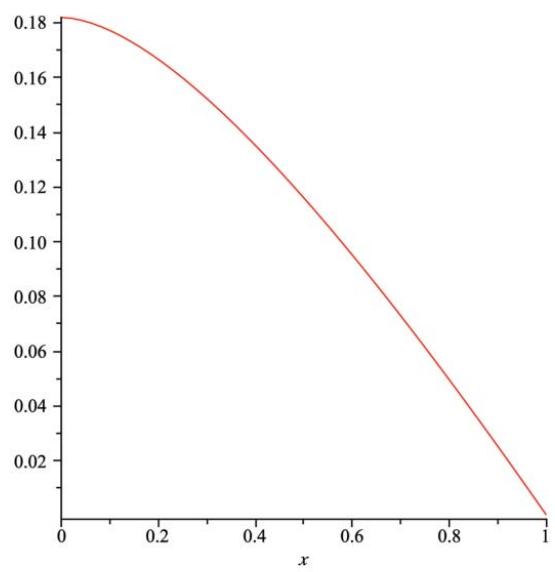

(c) $b_{2}(x)$ (asymptotic formula)

Figure 5. (a), (b) Degree distribution for $d=100, s=1.5$, and $\alpha=0.8$ as a function of age rank (rescaled); (c) $b_{2}(x)$ vs. the age rank of $v$ (rescaled) for the following set of parameters: $d=100, s=1.5$, and $\alpha=0.8$ (color figure available online).

have relatively large expected degree: most initial neighbors are sill in the network with high probability, and so the expected degree is close to $d$. In the worst position are middle-aged vertices, which are not old enough to attract new neighbors but have also had many initial neighbors disappear. The expected in-degree is, of course, a decreasing function: the older the vertex, the more time it has to accumulate a high degree. 
With Theorem 3.1 in hand, we now can easily derive a formula for $b_{2}\left(v_{i}\right)$. Suppose that $\operatorname{deg}^{-}\left(v_{i}, L\right) \geq \log ^{4} n$ (or $\operatorname{deg}\left(v_{i}, L\right) \geq \log ^{4} n$, since the out-degree is negligible) with $i=x n$, and the initial rank of $v_{i}$ is $R$. It follows from (5.2) that

$\mathbb{E}\left(\sum_{u \sim v_{i}} \operatorname{deg}^{-}(u, L)\right) \sim d(1-\alpha) 2^{\alpha}\left(\left(\frac{2 R}{n}\right)^{1-s}-1\right)^{-\alpha /(1-s)} x^{-\alpha} \int_{x}^{1} y^{\alpha} \cdot g(y) d y$.

Since the in-degree of $v_{i}$ is well concentrated around its expectation with extreme probability, we are ready to state the last result of this paper. Figure 5 (c) then presents the value of $b_{2}\left(v_{i}\right)$ based on the asymptotic formula.

Theorem 3.2. Let $0<\alpha<1, d \in \mathbb{N}, i=i(n) \in[n]$, and let $v_{i}$ be the vertex whose age rank at time $L$ equals $a_{L}\left(v_{i}\right)=i=x n$ and $\operatorname{deg}^{-}\left(v_{i}, L\right) \geq \log ^{4} n$. Then with extreme probability,

$$
b_{2}\left(v_{i}\right) \sim \frac{\mathbb{E}\left(\sum_{u \sim v_{i}} \operatorname{deg}^{-}(u, L)\right)}{\mathbb{E} \operatorname{deg}^{-}\left(v_{i}, L\right)} \sim \frac{1+\alpha}{1-x^{1+\alpha}} \int_{x}^{1} y^{\alpha} \cdot g(y) d y .
$$

\section{Conclusion}

This paper outlines a model of network self-organization that is driven by the ranks of individual nodes in terms of arbitrary attributes that are inherently individual phenomena. This model may be applied to a broad array of social systems in which nodal attributes (and rank) may represent wealth, power, beliefs, skills, or any other node-level variables that are likely to play an important role in networking behavior. This is a stochastic model involving the formation and deletion of network ties and adjustment in ranks, as nodes dynamically enter and exit the system over time.

This research builds on prior work in ranking and associated models of network self-organization, and continues the enterprise of linking these network models to enhance our understanding of the dynamics of real-world social systems. An important area for future research is to consider in detail how network structure evolves as a function of nodal attributes. Of course, this requires not only models of networks per se, but also models of attributes of individuals and how these attributes manifest themselves in network structure.

On the other hand, nodal attributes are often exceedingly difficult to observe and can be a limiting factor in our ability to study complex self-organizing networks. However, given realistic models of how network structure is driven by these attributes, it is possible to estimate individual attributes based on structure only. This research provides a platform for more research, emphasizing 
analytical proof that exploits the potential "reversibility" of mathematical models to infer latent variables that are crucial to the further development of network science.

\section{Appendix}

\section{I. Introduction to the Differential Equations Method}

The general setting that is used in the differential equations method [Wormald 99] is a sequence of random processes indexed by $n$ (which in our case, is the number of objects in a set $\left.V_{t}\right)$. The aim is to find properties of the random process in the limit as $n \rightarrow \infty$. The conclusion we aim for is that variables defined on a random process are well concentrated, which informally means that with high probability, they are very close to certain deterministic functions. These functions arise as the solution to a system of ordinary first-order differential equations. One of the important features of this approach is that the computation of the approximate behavior of processes is clearly separated from the proof that the approximation is correct.

To show that the random variables in a process usually approximate the solution of differential equations, we need to use large-deviation inequalities. These inequalities are often used to give an upper bound on the probability that a random variable deviates very far from its expected value. In a typical situation with a random process, the aim is to show that the random variable $Y_{t}$ of interest is sharply concentrated. In fact,

$$
Y_{t}-Y_{0}=\sum_{i=1}^{t}\left(Y_{i}-Y_{i-1}\right)
$$

If the differences $Y_{i}-Y_{i-1}$ are independent, then the Chernoff bound is very useful (see, for example, [Janson et al. 00, Theorem 2.8]).

Theorem 5.I. [Janson et al. 00] Let $X$ be a random variable that can be expressed as a sum $X=\sum_{i=1}^{n} X_{i}$ of independent random indicator variables, where $X_{i} \in$ $\operatorname{Be}\left(p_{i}\right)$ with (possibly) different $p_{i}=\mathbb{P}\left(X_{i}=1\right)=\mathbb{E} X_{i}$. Then the following hold for $t \geq 0$ :

$$
\begin{aligned}
& \mathbb{P}(X \geq \mathbb{E} X+t) \leq \exp \left(-\frac{t^{2}}{2(\mathbb{E} X+t / 3)}\right), \\
& \mathbb{P}(X \leq \mathbb{E} X-t) \leq \exp \left(-\frac{t^{2}}{2 \mathbb{E} X}\right)
\end{aligned}
$$


In particular, if $\varepsilon \leq 3 / 2$, then

$$
\mathbb{P}(|X-\mathbb{E} X| \geq \varepsilon \mathbb{E} X) \leq 2 \exp \left(-\frac{\varepsilon^{2} \mathbb{E} X}{3}\right) .
$$

When the differences are not independent but there is a large degree of independence, results can often be obtained by making use of analogous bounds given for martingales.

Definition 5.2. A martingale is a sequence $X_{0}, X_{1}, \ldots$ of random variables defined on a random process such that

$$
\mathbb{E}\left(X_{n+1} \mid X_{0}, X_{1}, \ldots, X_{n}\right)=X_{n}
$$

In most applications, the martingale satisfies the property that

$$
\mathbb{E}\left(X_{n+1} \mid X_{0}, X_{1}, \ldots, X_{n}\right)=\mathbb{E}\left(X_{n+1} \mid X_{n}\right)=X_{n}
$$

As a simple example, consider the following "random walk." Toss a coin $n$ times. Let $S_{n}$ be the difference between the number of heads and the number of tails after $n$ tosses. Then $S_{n}$ is a martingale. Indeed,

$$
\mathbb{E}\left(S_{n+1} \mid S_{n}\right)=S_{n}+\frac{1}{2} \cdot 1+\frac{1}{2} \cdot(-1)=S_{n} .
$$

Clearly, the expected value of $S_{n}$ is zero. Thus, it is natural to expect that $S_{n}$ stays relatively close to zero. The following well-known Hoeffding-Azuma inequality serves as a tool to investigate this.

Lemma 5.3. Let $X_{0}, X_{1}, \ldots$ be a martingale. Suppose that there exist constants $c_{k}>0$ such that

$$
\left|X_{k}-X_{k-1}\right| \leq c_{k}
$$

for each $k \leq n$. Then for every $t>0$,

$$
\begin{aligned}
& \mathbb{P}\left(X_{n} \geq \mathbb{E} X_{n}+t\right) \leq \exp \left(-\frac{t^{2}}{2 \sum_{k=1}^{n} c_{k}^{2}}\right), \\
& \mathbb{P}\left(X_{n} \leq \mathbb{E} X_{n}-t\right) \leq \exp \left(-\frac{t^{2}}{2 \sum_{k=1}^{n} c_{k}^{2}}\right) .
\end{aligned}
$$


This is often applied with $t$ growing much faster than $\sqrt{n}$ and the $c_{k}$ all small nonzero integers. In the martingale discussed above, $c_{k}=1$ for all $k$. Hence

$$
\mathbb{P}\left(\left|S_{n}\right| \geq \alpha \sqrt{n}\right) \leq 2 \exp \left(\frac{(\alpha \sqrt{n})^{2}}{2 n}\right)=2 \exp \left(\frac{\alpha^{2}}{2}\right),
$$

which is arbitrarily small for $\alpha$ large enough.

Finally, let us mention that the Hoeffding-Azuma inequality can be generalized in many ways: an analogous inequality holds for supermartingales $\left(\mathbb{E}\left(X_{n+1} \mid X_{n}\right) \leq X_{n}\right)$ as well as submartingales $\left(\mathbb{E}\left(X_{n+1} \mid X_{n}\right) \geq X_{n}\right)$. Our proofs use the supermartingale method of [Pittel et al. 96], as described in [Wormald 99, Corollary 4.1]. We will use the following useful lemma.

Lemma 5.4. Let $G_{0}, G_{1}, \ldots, G_{L}$ be a random process and $X_{t}$ a random variable determined by $G_{0}, G_{1}, \ldots, G_{t}, 0 \leq t \leq L$. Suppose that for some real $\beta$ and $\gamma$,

$$
\mathbb{E}\left(X_{t}-X_{t-1} \mid G_{0}, G_{1}, \ldots, G_{t-1}\right)<\beta
$$

and

$$
\left|X_{t}-X_{t-1}-\beta\right| \leq \gamma
$$

for $1 \leq t \leq L$. Then for all $\varepsilon>0$,

$\mathbb{P}\left(\right.$ for some $t$ with $\left.0 \leq t \leq L: X_{t}-X_{0} \geq t \beta+\varepsilon\right) \leq \exp \left(-\frac{\varepsilon^{2}}{2 L \gamma^{2}}\right)$.

\subsection{Proof of Theorem 2.3}

Proof of Theorem 2.3. The conditional expected change in $r_{t}(v)$ in time step $t+1$, conditional on object $v$ surviving until time $t+1$ and that $r_{t}(v) \leq n / 2$, is given by

$$
\mathbb{E}\left(r_{t+1}(v)-r_{t}(v) \mid G_{t}\right)=-\frac{r_{t}(v)-1}{n-1}+2^{s-1}\left(\frac{r_{t}(v)}{n}\right)^{s} .
$$

If we define a real function $z(x)$ to model the behavior of $r_{x n}(v) / n$, this suggests the differential equation

$$
z^{\prime}(x)=-z(x)+2^{s-1} z(x)^{s}
$$

with the initial condition $z(0)=R / n$. The general solution is

$$
z(x)=\frac{1}{2}\left(C e^{(s-1) x}+1\right)^{1 /(1-s)}, \quad C \in \mathbb{R},
$$


and the particular solution is

$$
z(x)=\frac{1}{2}\left(\left(\left(\frac{R}{n}\right)^{1-s}-1\right) e^{(s-1) x}+1\right)^{1 /(1-s)} .
$$

We need to transform $r_{t}(v)$ into something close to a martingale. Since the general solution can be written in the form

$$
\log \left(\left(\frac{1}{2 z(x)}\right)^{s-1}-1\right)-(s-1) x=C \in \mathbb{R},
$$

we should consider the following real-valued function:

$$
H(r, t)=\log \left(\left(\frac{n}{2 r_{t}}\right)^{s-1}-1\right)-(s-1) \frac{t}{n} .
$$

We hope that a good concentration for $H(r, t)$ implies good behavior for $r_{t}(v)$.

Clearly, we cannot control the process when the rank of $v$ drops below some small value (say $\sqrt{n} \log ^{2} n$ ). However, up to this point of the process, we should expect good concentration for these random variables. But how can we assume that the random variable is above the threshold when we are about to investigate its shape? The following concept, simple but very powerful, comes to the rescue. Define the stopping time as follows:

$$
T=\min \left\{t \geq 0: r_{t}(v)<\frac{1}{2} \sqrt{n} \log ^{2} n \vee r_{t}(v)>\frac{n}{2}-\frac{1}{2} \sqrt{n} \log ^{2} n \vee t=t_{f}\right\} .
$$

A stopping time is any random variable $T$ with values in $\{0,1, \ldots\} \cup\{\infty\}$ for which it can be determined whether $T=\hat{t}$ for a given time $\hat{t}$ from knowledge of the process up to and including time $\hat{t}$. The name can be misleading, since a process does not stop when it reaches a stopping time. The key observation is that if a supermartingale $\left(X_{i}\right)$ is stopped at a stopping time (that is, $\left(X_{i}\right)$ becomes static for all time after the stopping time), then the result is a supermartingale.

Let $\mathbf{w}_{t}=\left(r_{t}(v), t\right)$, and consider the sequence of random variables $\left(H\left(\mathbf{w}_{t}\right)\right.$ : $\left.0 \leq t \leq t_{f}\right)$. Let us stress again that $H$ is chosen so that $H(\mathbf{w})$ is close to a constant along every trajectory $\mathbf{w}$ of the differential equation. It is easy to check that the second-order partial derivatives of $H$ are $O\left(r^{-2}\right)=O\left(n^{-1} \log ^{-4} n\right)$ along the trajectory $\mathbf{w}_{t}$, provided that $T>t$. Therefore, with $i \wedge T$ denoting $\min \{i, T\}$ and using the fact that the rank changes by at most one in each step, we have

$$
\begin{aligned}
\left|H\left(\mathbf{w}_{(t+1) \wedge T}\right)-H\left(\mathbf{w}_{t \wedge T}\right)\right| & =O\left(\frac{1}{r_{t \wedge T}(v)}\right)=O\left(\frac{1}{\sqrt{n} \log ^{2} n}\right), \\
\mathbb{E}\left(H\left(\mathbf{w}_{(t+1) \wedge T}\right)-H\left(\mathbf{w}_{t \wedge T}\right) \mid G_{t \wedge T}\right) & =O\left(\frac{1}{n \log ^{4} n}\right) .
\end{aligned}
$$


Now we may apply Lemma 5.4 to the sequence $\left(H\left(\mathbf{w}_{t \wedge T}\right): 0 \leq t \leq\right.$ $\left.t_{f}\right)$, and symmetrically to $\left(-H\left(\mathbf{w}_{t \wedge T}\right): 0 \leq t \leq t_{f}\right)$, with $\varepsilon=1 / \log ^{1 / 2} n, \beta=$ $O\left(1 / n \log ^{4} n\right)$, and $\gamma_{t}=O\left(1 / \sqrt{n} \log ^{2} n\right)$, to show that with extreme probability,

$$
\left|H\left(\mathbf{w}_{t \wedge T}\right)-H\left(\mathbf{w}_{t_{0}}\right)\right|=O\left(\log ^{-1 / 2} n\right) .
$$

Since $H\left(\mathbf{w}_{0}\right)=\log \left((2 R / n)^{1-s}-1\right)$, it follows from the definition of $H$ that with extreme probability,

$$
\begin{aligned}
r_{t}(v) & =\frac{n}{2}\left(e^{H\left(\mathbf{w}_{0}\right)} e^{(s-1) t / n}+1\right)^{1 /(1-s)}\left(1+O\left(\log ^{-1 / 2} n\right)\right) \\
& =\frac{n}{2}\left(\left(\left(\frac{2 R}{n}\right)^{1-s}-1\right) e^{(s-1) t / n}+1\right)^{1 /(1-s)}\left(1+O\left(\log ^{-1 / 2} n\right)\right)
\end{aligned}
$$

for every $0 \leq t \leq T$.

To complete the proof, we need to show that with extreme probability, $T=t_{f}$. The events asserted by (2.1) hold with extreme probability up until time $T$, as shown above. Thus in particular, with extreme probability,

$$
r_{T}(v)>(1+o(1)) \sqrt{n} \log ^{2} n
$$

and

$$
r_{T}(v)<n / 2-(1+o(1)) \sqrt{n} \log ^{2} n,
$$

which implies that $T=t_{f}$ with extreme probability.

\subsection{Power Law for $s>1$}

Let $Z_{k}$ denote the number of vertices of degree $k$, and let $Z_{\geq k}=\sum_{l \geq k} Z_{l}$.

Theorem 5.5. Let $0<\alpha<1$ and $d \in \mathbb{N}, \log ^{4} n \leq k \leq n^{\alpha / 2} \log ^{-3 \alpha} n$. Then with extreme probability,

$$
Z_{\geq k}=(1+o(1))\left(\frac{d(1-\alpha)}{k(1+\alpha)}\right)^{1 / \alpha} n
$$

The proof is a consequence of the following result.

Theorem 5.6. Let $0<\alpha<1, d \in \mathbb{N}, i=i(n) \in[n]$, and let $v_{i}$ be the vertex whose age rank at time $L$ equals $a_{L}\left(v_{i}\right)=i=x n$. Let $R$ be the initial rank of $v_{i}$. 
(i) Assume that $\sqrt{n} \log ^{2} n<R<n / 2-\sqrt{n} \log ^{2} n$. Then the expected degree of $v_{i}$ is given by

$\mathbb{E} \operatorname{deg}\left(v_{i}, L\right)$

$$
=\left(1+O\left(\log ^{-1 / 2} n\right)\right) \frac{d(1-\alpha) 2^{\alpha}}{1+\alpha}\left(\left(\frac{2 R}{n}\right)^{1-s}-1\right)^{-\alpha /(1-s(}\left(x^{-\alpha}-x\right),
$$

provided that $x=o(1)$ or $R / n=o(1)$; otherwise, $\mathbb{E} \operatorname{deg}\left(v_{i}, L\right)=O(1)$.

(ii) Assume that $R>n / 2+\sqrt{n} \log ^{2} n$. Then $\mathbb{E} \operatorname{deg}\left(v_{i}, L\right)=O(1)$.

(iii) Moreover, if $\mathbb{E} \operatorname{deg}\left(v_{i}, L\right) \geq \log ^{4} n$, then with extreme probability,

$$
\operatorname{deg}\left(v_{i}, L\right)=\mathbb{E} \operatorname{deg}\left(v_{i}, L\right)+O\left(\sqrt{\mathbb{E} \operatorname{deg}\left(v_{i}, L\right)} \log n\right),
$$

and if $\mathbb{E} \operatorname{deg}\left(v_{i}, L\right)<\log ^{4} n$, then with extreme probability, $\operatorname{deg}\left(v_{i}, L\right)=$ $O\left(\log ^{4} n\right)$.

Proof. For (i), let us consider vertices $v_{i}$ and $v_{j}$ with respective age ranks $a_{L}\left(v_{i}\right)=i$ and $a_{L}\left(v_{j}\right)=j$, and let $i=x n$ and $j=y n(i<j)$. Suppose that $v_{i}$ obtained an initial rank of $R$. Let $t_{i}$ and $t_{j}$ be the respective times that vertices $v_{i}$ and $v_{j}$ were born. By Theorem 2.1,

$$
\begin{aligned}
& t_{i}=L-\left(1+O\left(\log ^{-1 / 2} n\right)\right) n \log \left(\frac{1}{x}\right), \\
& t_{j}=L-\left(1+O\left(\log ^{-1 / 2} n\right)\right) n \log \left(\frac{1}{y}\right),
\end{aligned}
$$

with extreme probability, and so $t_{j}-t_{i}=\left(1+O\left(\log ^{-1 / 2} n\right)\right) n \log (y / x)$. By Theorem 2.3, $v_{i}$ had with extreme probability the following rank when $v_{j}$ was born:

$$
r_{t_{j}}\left(v_{i}\right)=\frac{n}{2}\left(\left(\left(\frac{2 R}{n}\right)^{1-s}-1\right)\left(\frac{y}{x}\right)^{s-1}+1\right)^{1 /(1-s)}\left(1+O\left(\log ^{-1 / 2} n\right)\right) .
$$

Thus, the contribution to the degree of $v_{i}$ of vertices born after $v_{i}$ is the sum of independent indicator variables of the event that a vertex $v_{j}$ links to $v_{i}$ in a particular substep of time step $t_{j}$. The probability of this event is $r_{t_{j}}\left(v_{i}\right)^{-\alpha} / g_{\alpha}(n)$, where

$$
g_{\alpha}(n)=\sum_{j=1}^{n} j^{-\alpha}=\frac{n^{1-\alpha}}{1-\alpha}+O(1) .
$$

Since every vertex has initial degree $d$, the contribution to the degree of $v_{i}$ by older vertices is $O(d)$. We thus obtain the following expression for the expected 
degree:

$$
\begin{aligned}
\mathbb{E} \operatorname{deg}\left(v_{i}, L\right)= & O(d)+\left(1+O\left(\log ^{-1 / 2} n\right)\right) d(1-\alpha) 2^{\alpha} \\
& \times \int_{x}^{1}\left(\left(\left(\frac{2 R}{n}\right)^{1-s}-1\right)\left(\frac{y}{x}\right)^{s-1}+1\right)^{-\alpha /(1-s)} d y .
\end{aligned}
$$

If $x=\Omega(1)$ and $R / n=\Omega(1)$, then the expected degree is a constant, and the degree is smaller than $\log ^{2} n$ with extreme probability. Otherwise, it simplifies to

$$
\begin{aligned}
\mathbb{E} & \operatorname{deg}\left(v_{i}, L\right) \\
& =\left(1+O\left(\log ^{-1 / 2} n\right)\right) d(1-\alpha) 2^{\alpha}\left(\left(\frac{2 R}{n}\right)^{1-s}-1\right)^{-\alpha /(1-s)} x^{-\alpha} \int_{x}^{1} y^{\alpha} d y \\
& =\left(1+O\left(\log ^{-1 / 2} n\right)\right) \frac{d(1-\alpha) 2^{\alpha}}{1+\alpha}\left(\left(\frac{2 R}{n}\right)^{1-s}-1\right)^{-\alpha /(1-s)}\left(x^{-\alpha}-x\right),
\end{aligned}
$$

which finishes the proof of (i).

The proof of (ii) is easy: it follows from Theorem 2.3 that with extreme probability, $v_{i}$ has rank at least $n / 2$ during the whole process. Hence

$$
\mathbb{E} \operatorname{deg}\left(v_{i}, L\right) \leq n \cdot(1+o(1)) d(1-\alpha) \frac{(n / 2)^{-\alpha}}{n^{1-\alpha}}=O(1)
$$

Finally, in order to get (iii), it is enough to observe that if $\mathbb{E} \operatorname{deg}\left(v_{i}, L\right)=$ $\Omega\left(\log ^{4} n\right)$, then with extreme probability,

$$
\operatorname{deg}\left(v_{i}, L\right)=\mathbb{E} \operatorname{deg}\left(v_{i}, L\right)\left(1+O\left(\log ^{-1 / 2} n\right)\right),
$$

by the Chernoff bound.

As we already mentioned, at this point, Theorem 5.5 follows easily from the previous theorem. If $i=o\left(k^{-1 / \alpha} n\right)$, then with probability $1 / 2+o(1)$, vertex $v_{i}$ has degree at least $k$, but $o\left(k^{-1 / \alpha} n\right)$ vertices of this type turn out to be negligible compared to the total number of vertices of degree at least $k$. (Note that it follows from part (ii) that with probability $1 / 2+o(1)$, vertex $v_{i}$ gets initial rank at least $n / 2+\sqrt{n} \log ^{2} n$ and so has no chance to accumulate a large number of vertices, regardless of how old it is at time $L$.) Since the expected degree of $v_{i}$ is of order

$$
\left(\frac{R}{n} \cdot \frac{i}{n}\right)^{-\alpha}
$$

the probability that a vertex has degree at least $k$ is equal to $O\left(\left(k^{-1 / \alpha} n / i\right)^{s}\right)$. Hence, the expected number of vertices with $i \geq i_{0}=C k^{-1 / \alpha} n$ ( $C$ is a large 
constant) is equal to

$$
\sum_{i=i_{0}}^{n} O\left(\left(\frac{k^{-1 / \alpha} n}{i}\right)^{s}\right)=O\left(\left(k^{-1 / \alpha} n\right)^{s}\right) \cdot O\left(i_{0}^{1-s}\right)=O\left(C^{1-s} k^{-1 / \alpha} n\right) .
$$

This number again turns out to be negligible for $C \rightarrow \infty$ (that is, $i_{0} \gg k^{-1 / \alpha} n$ ).

Therefore, the only nontrivial probability of having degree at least $k$ is for vertices of age rank $i=c k^{-1 / \alpha} n$ for $c \in(0, \infty)$. The threshold $R_{0}=R_{0}(k, i)$ on the initial rank that causes the vertex to have degree at least $k$ at time $L$ is the following:

$$
R_{0}=\frac{n}{2}\left(\left(\frac{d(1-\alpha) 2^{\alpha}}{k(1+\alpha)}\left(\left(\frac{i}{n}\right)^{-\alpha}-\left(\frac{i}{n}\right)\right)\right)^{(1-s) / \alpha}+1\right)^{1 /(1-s)}
$$

To be precise, it follows from Theorem 5.5 that $R<\left(1-\log ^{-1 / 3} n\right) R_{0}$ implies that $\operatorname{deg}\left(v_{i}, L\right) \geq k$, whereas for $R>\left(1+\log ^{-1 / 3} n\right) R_{0}$, we get that $\operatorname{deg}\left(v_{i}, L\right)<$ $k$. Hence, the expected number of vertices of degree at least $k$ is

$$
\begin{aligned}
(1 & +o(1)) \sum_{i=1}^{n} F\left(R_{0} / n\right)=(1+o(1)) \sum_{i=1}^{n}\left(2 R_{0} / n\right)^{s} / 2 \\
& =\left(\frac{1}{2}+o(1)\right) \sum_{i=1}^{n}\left(\left(\frac{d(1-\alpha) 2^{\alpha}}{k(1+\alpha)}\left(\left(\frac{i}{n}\right)^{-\alpha}-\left(\frac{i}{n}\right)\right)\right)^{(1-s) / \alpha}+1\right)^{s /(1-s)} \\
& =\left(\frac{1}{2}+o(1)\right) n \int_{0}^{1}\left(\left(\frac{d(1-\alpha) 2^{\alpha}}{k(1+\alpha)}\left(x^{-\alpha}-x\right)\right)^{(1-s) / \alpha}+1\right)^{s /(1-s)} d x \\
& =\left(\frac{1}{2}+o(1)\right) n\left(\frac{d(1-\alpha) 2^{\alpha}}{k(1+\alpha)}\right)^{1 / \alpha}
\end{aligned}
$$

and the result follows from the Chernoff bound.

Acknowledgments. Paweł Prałat gratefully acknowledges support from MPrime and Ryerson University.

\section{References}

[Aiello 09] W. Aiello, A. Bonato, C. Cooper, J. Janssen, and P. Prałat. "A Spatial Web Graph Model with Local Influence Regions." Internet Mathematics 5 (2009), $175-196$.

[Barabási and Albert 99] A.-L. Barabási and R. Albert. "Emergence of Scaling in Random Networks." Science 286:5439 (1999), 509-512. 
[Bonato 08] A. Bonato. A Course on the Web Graph, American Mathematical Society Graduate Studies Series in Mathematics. AMS, 2008.

[Chung and Lu 04] F. R. K. Chung and L. Lu. Complex Graphs and Networks. American Mathematical Society, 2004.

[Fortunato et al. 06] S. Fortunato, A. Flammini, and F. Menczer. "Scale-Free Network Growth by Ranking." Phys. Rev. Lett. 96:21 (2006), 218701.

[Henry and Prałat 11] A. Henry and P. Prałat. "Rank-Based Models of Network Structure and the Discovery of Content." In Proceedings of the 8th Workshop on Algorithms and Models for the Web Graph (WAW 2011), Lecture Notes in Computer Science 6732, pp. 62-73. Springer, 2011.

[Henry et al. 11] A. D. Henry, P. Prałat, and C. Q. Zhang. "Emergence of Segregation in Evolving Social Networks." Proceedings of the National Academy of Sciences 108:21 (2011), 8605-8610.

[Jackson 08] M. O. Jackson. Social and Economic Networks. Princeton University Press, 2008.

[Janson et al. 00] S. Janson, T. Łuczak, and A. Ruciński. Random Graphs. Wiley, 2000.

[Janssen and Prałat 09] J. Janssen and P. Prałat. "Protean Graphs with a Variety of Ranking Schemes." Theoretical Computer Science 410 (2009), 5491-5504.

[Janssen and Prałat 10] J. Janssen and P. Prałat. "Rank-Based Attachment Leads to Power Law Graphs." SIAM Journal on Discrete Mathematics 24 (2010), 420-440.

[Janssen et al. 10] J. Janssen, P. Prałat, and R. Wilson. "Estimating Node Similarity from Co-citation in a Spatial Graph Model." In Proceedings of the 2010 ACM Symposium on Applied Computing (SAC)—Special Track on Self-Organizing Complex Systems, pp. 1329-1333, 2010.

[Lazer 01] D. Lazer. "The Coevolution of Individual and Network." Journal of Mathematical Sociology 25:1 (2001), 69-108.

[Luczak and Prałat 06] T. Luczak and P. Prałat. "Protean Graphs." Internet Mathematics 3 (2006), 21-40.

[Newman 03] M. E. J. Newman. "The Structure and Function of Complex Networks." SIAM Review 45:2 (2003), 167-256.

[Pittel et al. 96] B. Pittel, J. Spencer, and N. Wormald. "Sudden Emergence of a Giant k-Core in a Random Graph." J. Combinatorial Theory, Series B 67 (1996), 111-151.

[Prałat 08] P. Prałat. "A Note on the Diameter of Protean Graphs." Discrete Mathematics 308 (2008), 3399-3406.

[Prałat 11] P. Prałat. "Connectivity Threshold and Recovery Time in Rank-Based Models for Complex Networks." Discrete Mathematics 311 (2011), 932-939.

[Prałat and Wormald 09] P. Prałat and N. Wormald. "Growing Protean Graphs." Internet Mathematics 4 (2009), 1-16.

[Wasserman and Faust 94] S. Wasserman and K. Faust. Social Network Analysis: Methods and Applications. Cambridge University Press, 1994.

[Wormald 99] N. Wormald. "The Differential Equation Method for Random Graph Processes and Greedy Algorithms." In Lectures on Approximation and Randomized 
Algorithms, edited by M. Karoński and H. J. Prömel, pp. 73-155. Warsaw: PWN, 1999.

[Wormald 04] N. C. Wormald. "Random Graphs and Asymptotics." In Handbook of Graph Theory, edited by J. L. Gross and J. Yellen. pp. 817-836. CRC, 2004.

Adam Douglas Henry, School of Government and Public Policy, University of Arizona, Tucson, AZ 85721-0027 (adhenry@email.arizona.edu)

Paweł Prałat, Department of Mathematics, Ryerson University, Toronto, ON, Canada, M5B 2K3 (pralat@ryerson.ca) 